\title{
NUEVAS ESPECIES DE LA TRIBU SPERMACOCEAE (RUBIACEAE) PARA LA FLORA DE BRASIL
}

\author{
Elsa L. Cabral ${ }^{1}$ \\ Nélida M. Bacigalupo²
}

Recebido em 11/9/96. Aceito em 12/6/97

RESUMEN - (Nuevas especies de la tribu Spermacoceae (Rubiaceae) para la flora de Brasil). Se describen e ilustran cuatro especies nuevas para la flora de Brasil: Borreria paulista del Estado de São Paulo, B. rosmariinifolia del Estado de Minas Gerais, Mitracarpus steyermarkii y Psyllocarpus intermedius del Estado de Bahia.

Palabras-clave: Rubiaceae, Spermacoceae, especies nuevas

ABSTRACT - (New species of the tribe Spermacoceae (Rubiaceae) for the Brazil flora). Four new species of the tribe Spermacoceae (Rubiaceae), for Brazil are described and illustrated: Borreria paulista (São Paulo), B. rosmariinifolia (Minas Gerais), Mitracarpus steyermarkii and Psyllocarpus intermedius (Bahia).

Key words: Rubiaceae, Spermacoceae, new species

\section{Introducción}

Durante el transcurso del estudio de los géneros sudamericanos de la tribu Spermacoceae, actualmente en realización, se han encontrado cuatro novedades para la flora de Brasil que se dan a conocer a continuación.

Se trata de dos especies del género Borreria G.F.W. Mey., sect. Borreria (Bacigalupo \& Cabral 1996) que se caracterizan por poseer frutos de dos mericarpos, a la madurez separados casi totalmente desde el ápice y dehiscentes longitudinalmente por la línea media ventral, flores de estambres y estilo exertos, estilos capitadobilobados y semillas finamente reticulado-escrobiculadas. Una de las novedades, $B$.

\footnotetext{
' Facultad de Ciencias Exactas, Físicas y Naturales y Agrimensura (UNNE), Instituto de Botánica del Nordeste, C.C. 209, 3400 Corrientes, Argentina

${ }^{2}$ Instituto de Botánica Darwinion, C.C. 22, 1642 San Isidro, Buenos Aires, Argentina
} 
paulista, ha sido encontrada en el Estado de São Paulo y la otra, B. rosmariinifolia en Minas Gerais.

A estas hay que sumar una especie del género Mitracarpus Zucc., caracterizado por sus flores hipocraterimorfas, y sobre todo por sus frutos de dehiscencia transversal, con una mitad superior caediza y una inferior persistente. Se trata de M. steyermarkii recolectada en el Estado de Bahia. Por último, la cuarta novedad corresponde al género Psyllocarpus, género endémico de Brasil, el cual se reconoce por los frutos de 2 mericarpos de dehiscencia septífraga con el tabique interlocular persistente entre las dos valvas del fruto. $P$. intermedius no presenta, como las restantes especies del género, los frutos fuertemente comprimidos dorsiventralmente y participa de algunos caracteres de las dos secciones del género establecidas por Kirkbride (1979).

\section{Material y métodos}

Este estudio se ha realizado con material de los herbarios nacionales y extranjeros cuyas siglas se registran de acuerdo con Holmgren et al. (1990).

\section{Resultados y discusión}

Borreria paulista E.L.Cabral et Bacigalupo sp. nov.

Figura 1.

Suffrutex $10-16 \mathrm{~cm}$ altus, ramis prostratis, radicantibus, subtetragonis, villosis. Folia pseudoverticillata, sessilia, $5-12 \mathrm{~mm}$ longa, $1-2 \mathrm{~mm}$ lata, anguste ovata, apice acuto vel acuminato, basi attenuata, in superficie glabra vel pubescentia, a tergo et nervo glabra vel subpilosa, rhaphidibus laetis. Glomeruli 1-4 in quoque ramo, $5-8 \mathrm{~mm}$ diam., 4-6 bracteis aequantibus maioribusve. Calyx 4-partitus, segmentis linearibus lanceolatis, 1-2mm longis, scabridiusculis. Corolla $1,5-3,5 \mathrm{~mm}$ longa, lobulis tam longis quam tubo. Stamina exerta, filis $1,5 \mathrm{~mm}$ longis et antheris $1 \mathrm{~mm}$ longis. Stylus 1,5-3mm longus, stigmate capitato-bilobato. Capsula 1,6-2,5mm longa, in tertio apicali pilosa. Semina $2-2,5 \mathrm{~mm}$ longa.

Holotipo: BRASIL. São Paulo, Município de Itirapina, Itirapina, Rio Claro, 5 $\mathrm{km}$ da rodovia Washington Luiz na estrada, flor branca, planta em cerrado, 11/IV/ 1962, I.M. Válio 231 (SP). Isotipo (SP).

Paratipos: BRASIL. São Paulo, Município de Itirapina, 30/IV/1923, G. Gehrt 8341 (CTES, SP); São Carlos, $10 \mathrm{~km}$ E da cidade de Itirapina, 4/IV/1962, I. Mimura 354 (SP); Região Perimetral da Represa do Lobo, 9/X/1973, Ma. H. Souza s/n (SPF 79777); Itirapina, Estrada da Ubá, 11/IL/1993, Fabio de Barros 2643 (SP).

Sufrútice muy ramoso, de $10-16 \mathrm{~cm}$ alt., de ramas postradas o decumbentes, 


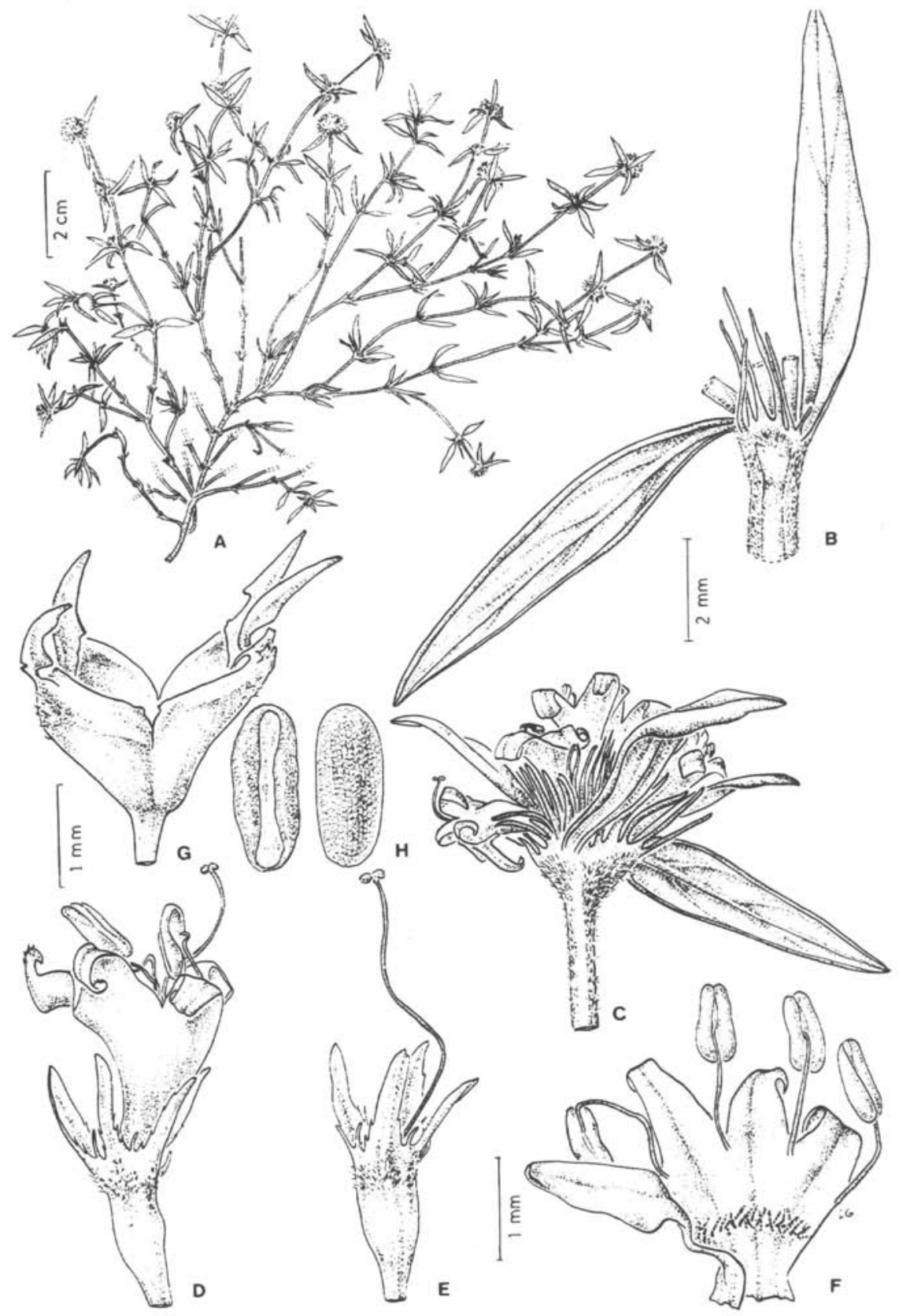

Figura 1. Borreria paulista Cabral \& Bacigalupo. A: planta; B: nudo foliar; C: glomérulo; D: flor; E: hipanti, cáliz y estilo; F: corola desplegada; G: fruto; H: semilla, cara ventral y dorsal. A-F (Valio 231-SP 64042); G-H (Gehrt 8341). 
radicantes, subtetrágonas, vellosas. Hojas pseudo-verticiladas, sésiles, de $5-12 \mathrm{~mm}$ long. x 1-2mm lat., angostamente ovadas, de ápice agudo o acuminado y base atenuada, glabras o subglabras, casi siempre con pelos visibles sobre ambas caras en la base foliar, margen glabro, con puntos brillantes (rafidios) en el haz. Vaina estipular de $1 \mathrm{~mm}$ long., villosa, hasta con 6-7 lacinias glabras, de 1,5-2mm long. Glomérulos 1-4 por rama, de 5-8mm diám., con 4-6 brácteas iguales o mayores que éstos. Cáliz 4partido, de segmentos linear-lanceolados, de 1-2mm long., glabros con algunos pelitos sobre la mitad basal del margen, y diminutos dientes intercalares en los senos intercalares. Corola blanca, infundibulifome de 2,5-3,5mm long, de lóbulos tan largos como el tubo, solo con pelos dispersos en el dorso o en el ápice de los lóbulos y un anillo de pelos moniliformes en su interior, a la mitad del tubo corolino. Estambres exertos, filamentos de 1,5-2,2 mm long. y anteras de 0,8-1 mm long. Estilo de 1,5-3mm long., estigma capitado-bilobado. Hipanto de 1-1,5mm long., piloso en el tercio superior. Cápsula de 1,6-2,5mm long.,pilosa en el tercio apical. Semillas de 1,5-2,5mm long. elipsoidales, finamente escrobiculadas, con estrofíolo sobre el surco ventral.

Distribución geográfica: Todo el material estudiado procede del Brasil, del Estado de São Paulo.

Borreira paulista es afin a B. quadrifaria Cabral (1986) por sus tallos ramificados y sus hojas seudoverticiladas y el cáliz 4-partido pero se diferencia por tener esta última tallos erectos escasamente ramificados con hojas de $15-30(-50) \mathrm{mm}$ long. x 2,5$6 \mathrm{~mm}$ lat. y corolas de 4,2-4,5mm.

Borreria rosmariinifolia E.L.Cabral et Bacigalupo sp. nov.

Figura 2

Suffrutex $10-30 \mathrm{~cm}$ altus, paucis ramis basalibus, tetragonis, $1 \mathrm{~mm}$ latis, pauci vel nulliramificantibus, internodiis $5-15 \mathrm{~mm}$ longis, pilis hirtis. Folia pseudoverticillata, linearia, rigida, obliqua, 5-20 mm longa, 0,5-1 mm lata, interdum margine revoluto, raphidibus ateris. Glomeruli apicales, raro subapicales, $5-10 \mathrm{~mm}$ diam., bracteis 4-8 involucralibus rigidis, glomerulis duplo longioribus. Calyx 4-partitus, segmentis linearibus 1,5-2 mm longis, scabridiusculis. Corolla 3-3,5mm longa, lobulis tam longis quam tubo. Stamina exerta, filis antherisque $1 \mathrm{~mm}$ longis. Stylus $3-3,5 \mathrm{~mm}$ longus, stigmate capitato-bilobato. Capsula 2-2,5mm longa. Semen 1-1,5mm longum, scrobiculatum.

Holotipo: BRASIL. Minas Gerais. Municipio Santana do Riacho, Serra da Lapinha, Maciço NW da Serra do Cipó, próximo da localidade da Lapinha, ca. 50 km da Rodovia Belo Horizonte-Conceição do Mato Dentro. Subarbusto en solo arenopedregoso. Flores alvas, 27/III/1991, J. R. Pirani, A. M. Giuletti, N. Menezes, N. Meguro, J. Coffani-Nunes, F. Vitta, L. Parra-Lazzari 12168 (SPF) Isotipo: CTES.

Paratipos: BRASIL. Minas Gerais, Município de Jaboticatubas, km 115 ao 


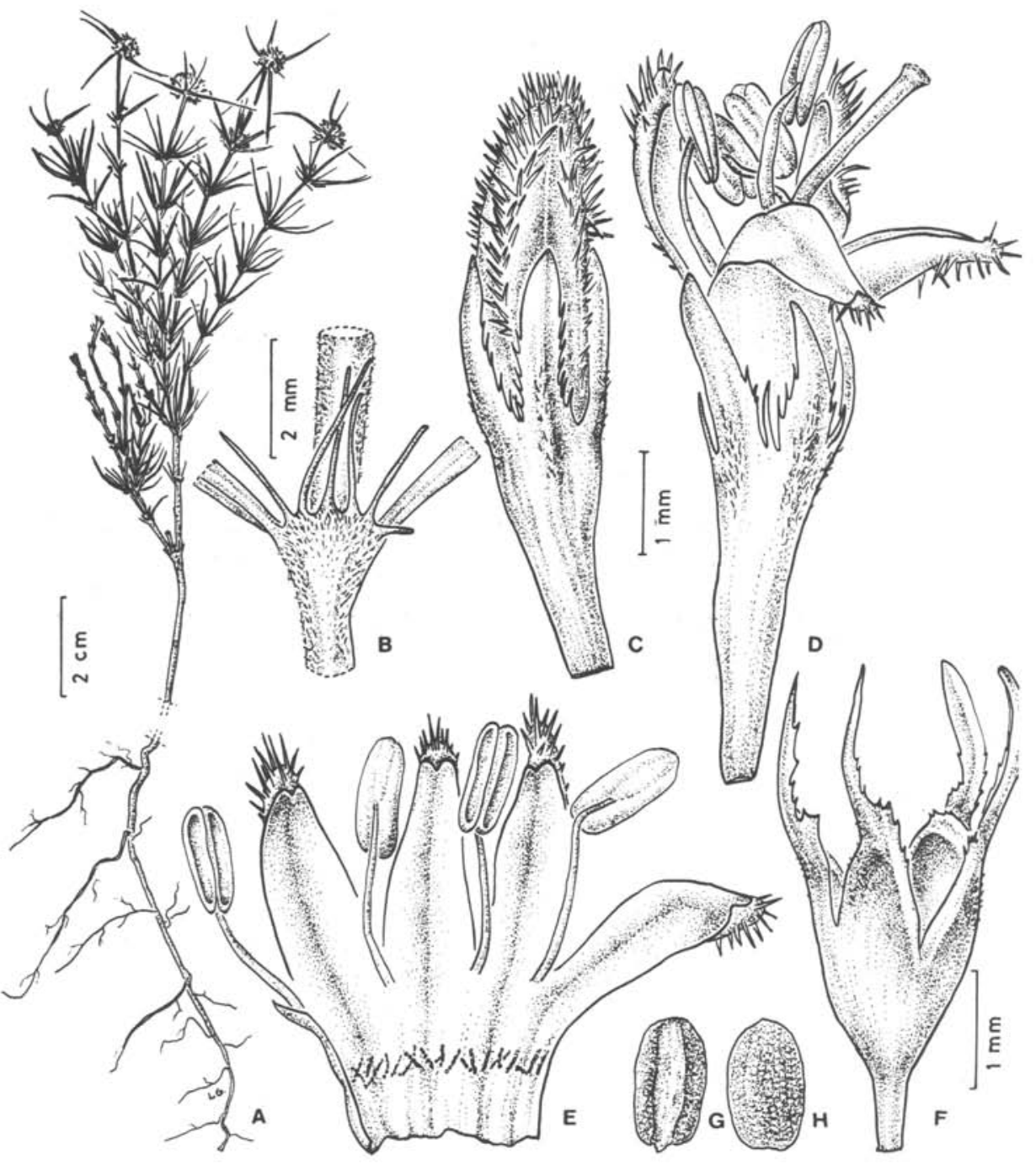

Figura 2. Borreria rosmariniifolia Cabral \& Bacigalupo. A: planta; B: vaina estípula; C: bóton floral; D: flor; E: corola desplegada; F: fruto; G y H: semilla, cara ventral y dorsal. A-H (Pirani et al. 12168). 
longo da rodovia Lagoa Santa-Conceição do Mato Dentro, Diamantina, 4/III/1972, A. B. Joly et al. 872 (SP); idem, A. B. Joly et al. 830 (SP); Município De Jaboticatubas, 29/V/1972, A. B. Joly et al. 2506 (SP). Município de Santana do Riacho, km 110 ao longo da rodovia Belo Horizonte-Conceição do Mato Dentro, 19/III/1983, M. Kawasaki 9117 (SPF); Serra do Cipó, km 115 (ca. $140 \mathrm{~km} \mathrm{~N}$ of Belo Horizonte), elev. 1250, 19/II/1968, H. Irwin 20469 (NY), 15-II-1968, H. Irwin 20131 (NY).

Sufrútice de 10-30cm alt., con 1-6 ramas basales, tetrágonas, de $1 \mathrm{~mm}$ lat., poco o no ramificadas, de entrenudos de 5-15mm long., hírtulos. Hojas seudo-verticiladas, lineares, rígidas, oblicuas, de $5-20 \mathrm{~mm}$ long. $x \quad 0,5-1 \mathrm{~mm}$ lat., a veces con borde revoluto, con rafidios manifiestos como puntitos negros. Vaina estipular de $0,5-1 \mathrm{~mm}$ long., pubérula a pubescente, con 3-5 lacinias glabras, de 1-3mm long. Glomérulos apicales, raro alguno subapical, de $5-10 \mathrm{~mm}$ diám., con 4-8 brácteas involucrales rígidas, dos veces más largas que los glomérulos. Alabastros con pelos hirtos. Cáliz 4partido, de segmentos lineares de 1,5-2mm long., escabriúsculos, con diminutos dientes intercalares. Corola, infundibuliforme de 3-3,5mm long., de lóbulos tan largos como el tubo, con pelos hirtos sobre la línea media dorsal de los lóbulos, pelos más largos hacia el ápice y anillo de pelos moniliformes cerca de la base en el interior del tubo corolino. Estambres exertos, filamentos y anteras de $1 \mathrm{~mm}$ long. Estilo de 3$3,5 \mathrm{~mm}$ long., estigma capitado-bilobado. Hipanto de $1-1,5 \mathrm{~mm}$, pubescente en el tercio superior. Cápsula de 2-2,5mm long., pubescente en el tercio superior. Semillas de 1$1,5 \mathrm{~mm}$ long., escrobiculada, con estrofíolo sobre el surco ventral.

Distribución geográfica: Todos los ejemplares estudiados proceden de Brasil, del Estado de Minas Gerais.

Borreira rosmariinifolia vive en suelo arenoso-rocoso, ca. 1200-1300 m. s. m. Es fácil reconocerla por sus hojas lineares similares a las de Rosmarinus officinalis $\mathrm{L}$. (romero) orientadas hacia el ápice, y por sus brácteas involucrales rígidas, patentes.

Mitracarpus steyermarkii E. L.Cabral et Bacigalupo, sp. nov. Figura 3.

Herba annua $9-15 \mathrm{~cm}$ alt., ramis erectis vel extensis, basi lignosis, subtetragonis vel teretibus, pubescentibus. Folia coriacea, sessilia, 10-25mm longa, 4-8mm lata, lanceolata, basi obtusa vel attenuata, apiculata, marginata, uninervia, irregulariter pilosa super nervo medio in tergo et margine basali, residuum glabrum. Glomeruli apicales $10-25 \mathrm{~mm}$ diam., bracteis 4-8 foliaceis. Calyx 4-partitus, segmentis linearlanceolatis, margine scabridiusculo, duobus longioribus, $2-3 \mathrm{~mm}$ longis. Corolla $7 \mathrm{~mm}$ longa, extra glabra. Antherae $0,5-0,7 \mathrm{~mm}$ longae. Stylus $5 \mathrm{~mm}$ longus. Flores adulti, pauli breviores, eodem glomerulo mixti (corolla $5,5 \mathrm{~mm}$, stylus $1 \mathrm{~mm}$ et antherae 1$1,5 \mathrm{~mm})$. Fructus non visus. 


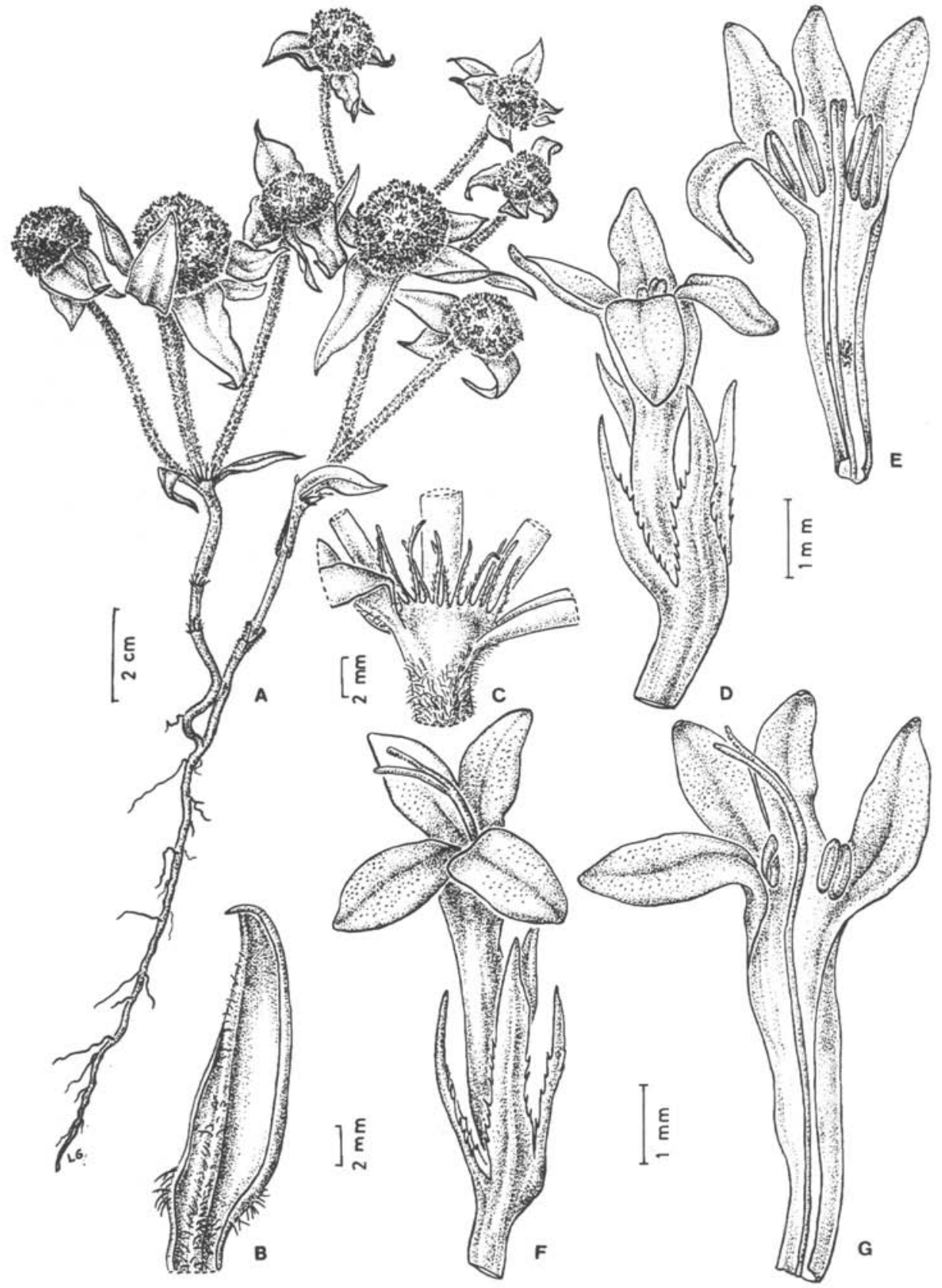

Figura 3. Mitracarpus steyermarkii Cabral \& Bacigalupo. A: planta; B: hoja, cara abaxial; C: vaina estipular; D-E: flor y su corola abierta e estilo; F-G: flor y su corola abierta y estilo. A-G (Krapovickas et al. 45331). 
Holotipo. BRASIL, Bahia, cerrado, $7 \mathrm{~km} \mathrm{~S}$ of Rio Piau, ca. $150 \mathrm{~km} \mathrm{SW}$ of Barreiras, 850 m. s.m., herb. to ca. $15 \mathrm{~cm}$ tall, with firm leathery leaves, corola white, 13/IV/1966, H. Irwin et al. 14690 (K).

Paratipo. BRASIL. Bahia, BR-020, km 336, 30-III-1983, A. Krapovickas, J. Valls, R. Veiga y G. Silva 38694 (CTES).

Hierba anual, de $9-15 \mathrm{~cm}$ alt., de ramas erguidas o extendidas, de base leñosa, subtetrágonas a cilíndricas, pubescentes. Hojas coriáceas, sésiles, de 10-25mm long. x 4-8mm lat., lanceoladas, de base obtusa o atenuada, apiculadas, marginadas, uninervias, irregularmente pilosas sobre el nervio medio en el envés y margen basal, el resto glabras. Vaina estipular de 3-4mm long., glabrescente, con 6-12 lacinias hírtulas, de 3-5 $\mathrm{mm}$ long. Glomérulos apicales de 10-25mm diám., con 4-8 brácteas foliáceas. Cáliz 4-partido, de segmentos linear-lanceolados, de borde escabriúsculo, 2 más largos, de 2-3mm long., alternando con los otros 2 ligeramente menores. Corola blanca, hipocrateriforme, de $7 \mathrm{~mm}$ long., exteriormente glabra y en su interior con los lóbulos micropapilados y un anillo de pelos cerca de la base del tubo. Anteras de 0,5$0,7 \mathrm{~mm}$ long. Estilo de $5 \mathrm{~mm}$ long. Hipanto de $1 \mathrm{~mm}$ long. Entremezcladas en el mismo glomérulo flores también maduras, algo menores, con corola $(5,5 \mathrm{~mm})$ y estilo menores y anteras de $1-1,5 \mathrm{~mm}$. Fruto no visto.

Distribución geográfica. Se conoce solo del Estado de Bahia. Crece en el campo.

Mitracarpus steyermarkii es afín a Mitracarpus megapotamicus(Sprengel) Kuntze, pero ésta es un sufrútice de mayor tamaño y más ramificado, las hojas son seudopecioladas, elípticas y totalmente hírtulas de 7-35mm long.

Psyllocarpus intermedius E. L. Cabral et Bacigalupo sp. nov. Figura 4

Suffrutex $50 \mathrm{~cm}$, ramificatissimus, ramis lateralibus gracilibus, glabris. Folia sessilia, linearia, 10-25mm longa, 0,5 mm lata, glabra, uninervia, margine revoluto. Glomeruli apicales 5-10 mm diam., bracteis 2-4 involucralibus, linearibus, longioribus quam glomerulis. Calyx 4-partitus, segmentis subulatis. 0,75-1,25mm longis. Corolla alba, glabra, 2,5-3,5mm longa, antheris 1-1,25mm longis. Stylus exertus, 2,5-3mm longus, bifidus. Capsula subglobosa, ambitus subelliptico, 2,5-3,5mm longo, glabra. Semina $2 \mathrm{~mm}$ longa.

Holotipo. BRASIL. Bahia, Município Mucuge, Barriguda, 2-3 km da rodovia para Palmeiras, ereta, flores alvas, campo rupestre, alt. 1200 m. s. m., 9/IV/1992, G. Hatschbach et al. 56892 (MBM). Isotipo (CTES, SI). 


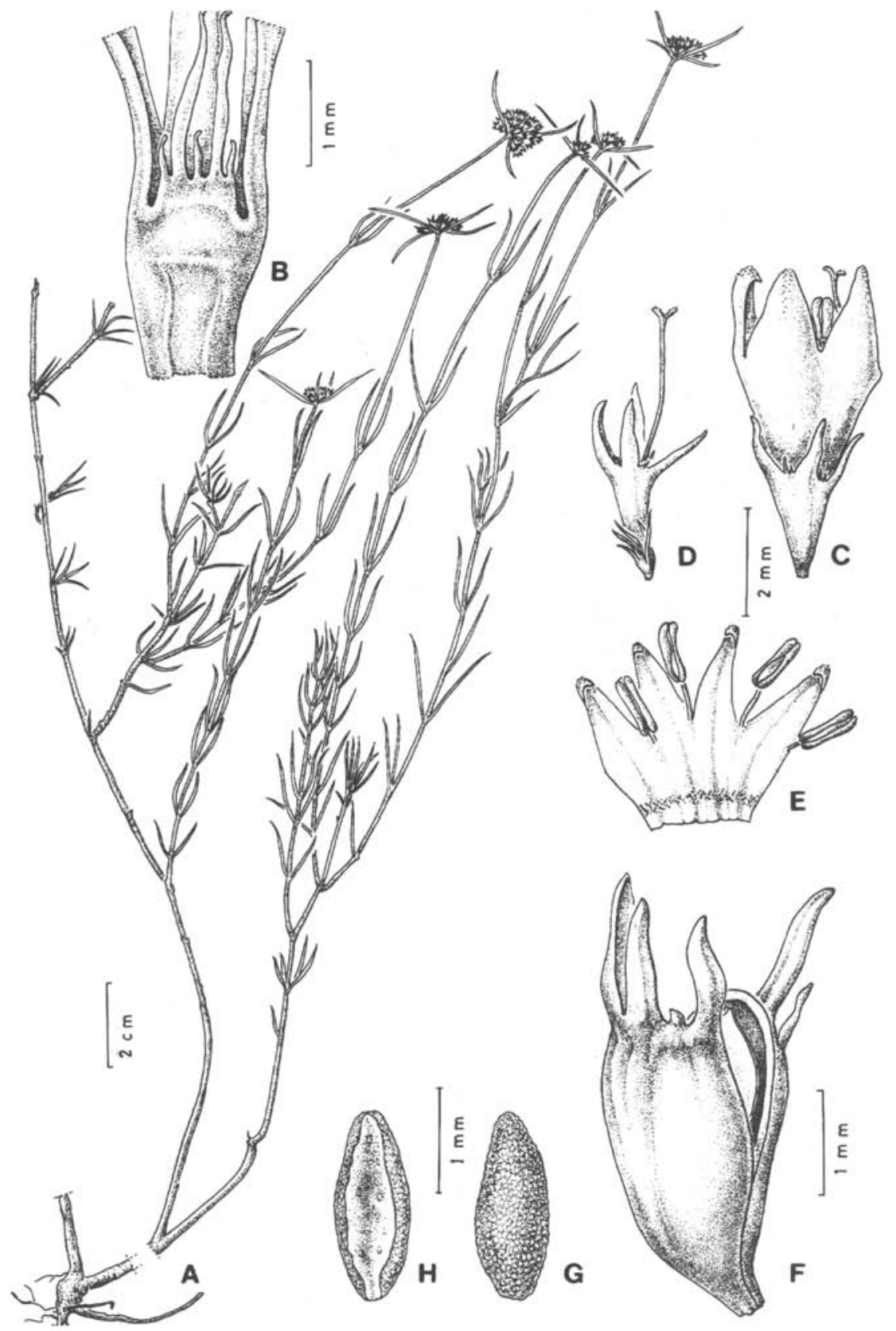

Figura 4. Psyllocarpus intermedius Cabral \& Bacigalupo. A: planta; B: vaina estipular; C: flor; D: hipanto, cáliz y estilo; E: corola desplegada; F: fruto; G-H: semilla, cara dorsal y ventral. (A-H, Hatschbach 56892). 
Sufrútice de $50 \mathrm{~cm}$ alt., glabro, muy ramificado, con numerosas ramas laterales, gráciles. Hojas sésiles, de 10-25mm long. x 0,5mm lat., lineares, subcilíndricas, ligeramente aplanadas dorsiventralmente, levemente surcadas a ambos lados del nervio central en la cara abaxial. Vaina estipular, 0,75-1,25mm long, con 3-5 lacinias de 0,5-1,25mm. Glomérulos apicales, de 5-10 mm de diám., con 2-4 brácteas involucrales, lineares, más largas que los glomérulos. Cáliz 4-partido, con segmentos de $0,75-2 \mathrm{~mm}$ long. y diminutos dientes intercalares. Corola blanca, micropapilada, de 2,5-3,5mm long., con lobulos iguales o más largos que el tubo, en su interior, cerca de la base con anillo ralo de pelos moniliformes. Estambres 4, exertos, de filamentos papilosos, ligeramente desiguales, 2 más largos, anteras de 1-1,25mm long. Granos de polen colporados, tectado-perforados, espinulados. Estilo exerto de 2,5-3mm long., bífido. Disco bipartido. Hipanto de 1,25-1,50mm long. Cápsula subglobosa, de contorno subelíptico, septífraga, de 2,5-3,5mm long., coronada por los sépalos. Semillas elipsoides de $1,5-1,7 \mathrm{~mm}$ long.

Kirkbride (1979) en la revisión del género Psyllocarpus separa a las especies en dos secciones: Sect. Psyllocarpus y Sect. Amazonica. P. intermedius presenta caracteres particulares que no se corresponden plenamente con ninguna de las dos secciones. A continuación se resumen las analogías y diferencias con cada una de las secciones establecidas por Kirkbride.

\begin{tabular}{|c|c|c|}
\hline Sect. Psyllocarpus & Psyllocarpus intermedius & Sect. Amazonica \\
\hline $\begin{array}{l}\text { Hojas cilíndricas, } \\
0.1-0.5(-1) \mathrm{mm} \text { lat. }\end{array}$ & $\begin{array}{l}\text { Hojas subcilíndricas de márge- } \\
\text { nes recurvos } 0,5 \mathrm{~mm} \text { lat. }\end{array}$ & Hojas planas $1,5-10 \mathrm{~mm}$ lat. \\
\hline $\begin{array}{l}\text { Flores isomorfas (Estambres } \\
\text { y estilo inclusos) }\end{array}$ & $\begin{array}{l}\text { Flores isomorfas (Estambres } \\
\text { y estilo exertos) }\end{array}$ & $\begin{array}{l}\text { Flores dimorfas (Estambres in- } \\
\text { clusos y estilo exerto o estambre } \\
\text { exerto y estilo incluso) }\end{array}$ \\
\hline Cáliz 2-partido & Cáliz 4-partido & Cáliz 4-partido \\
\hline Estigma capitado-bilobado & Estigma bífido & Estigma bífido \\
\hline $\begin{array}{l}\text { Granos de polen colpozonorados, } \\
\text { perforados }\end{array}$ & $\begin{array}{l}\text { Granos de polen colporados, } \\
\text { tectado-perforados, espinulados }\end{array}$ & $\begin{array}{l}\text { Granos de polen colporados, tec- } \\
\text { tado-espinulados }\end{array}$ \\
\hline Fruto comprimido & Fruto globoso & Fruto comprimido \\
\hline
\end{tabular}

\section{Referencias bibliográficas}

Bacigalupo,N.M. \& Cabral,E.L. 1996. Infrageneric classification of Borreria (Rubiaceae-Spermacoceae) on the basis of American species. Opera Botanica Belg. 7: 297-308.

Cabral E.L. 1986. Contribución al estudio del género Borreria sección Borreria (Rubiaceae). Parodiana 4(1): 133-142.

Holmgren,P.K. \& al. 1990. Index Herbariorum, Part I. Ed. 8. Regnum Veg. 120: 629 p.

Kirkbride, J.H., 1979. Revision of the genus Psyllocarpus (Rubiaceae). Smithsonian Contributions to Botany 41: 1-32

Schumann, K. 1888. Rubiaceae-Spermacoceae in Martius, Flora Brasiliensis 6 (6): 6-398; 401-409. 